\title{
Geographical and climatic effects on precipitation and pan evaporation
}

\author{
Chang-Soo Rim* \\ Department of Civil Engineering, Kyonggi University, San 94-6, lui-dong, Yeongtong-gu, Suwon-si, Kyonggi-do, \\ 443-760 South Korea
}

\begin{abstract}
Multiple linear regression (MLR) analyses and Mann-Kendall trend analyses were conducted in order to identify the importance of geographical factors (elevation, freshwater area, urbanization, and proximity to coast) and climatic factors (temperature, wind speed, relative humidity, and solar radiation) on the long-term averages and trends of precipitation and pan evaporation. Annual climate data and monthly climate data for January, April, July, and October, from 1973 to 2006, were collected from 54 weather observatories nationwide in South Korea in consideration of seasonal effects. GIS analyses were used to determine geographical characteristics in the 54 study areas, and land cover data were analyzed to determine the degree of urbanization. According to standard MLR analyses, proximity to coast had the most significant effect on annual and monthly precipitation. Accordingly, land areas that were farther inland experienced less precipitation, except in July, when the reverse was true. Except in April and July, proximity to coast had a greater effect on annual and monthly pan evaporation, and land areas that were farther inland experienced less pan evaporation. Average elevation had a greater effect than other geographical factors on annual and monthly precipitation trends. With regard to the annual pan evaporation trend, proximity to coast was the most important factor; however, the monthly pan evaporation trend was affected differently by geographical factors, depending on the season.
\end{abstract}

KEY WORDS: Precipitation · Pan evaporation · GIS · Multiple linear regression · Trend analysis

\section{INTRODUCTION}

Climatic change caused by global warming can affect hydrological circulation processes such as precipitation and evaporation; however, recent studies have found that geographical characteristics can affect precipitation and evaporation as well (IPCC 2007). Geographical characteristics have close relationships with convective precipitation in the summer, and the occurrence of convective precipitation in the summer is closely related to land moisture on the surface of the earth, conditions of vegetation, and topography.

Different types of landscape variability, such as urbanization, topography, land/sea contrasts, land surface moisture, and vegetation, can significantly affect the boundary layer and the cloud structure by altering the partitioning of the turbulent sensible and latent heat fluxes, and moisture may be added to the bound- ary layer through evaporation (Collins \& Avissar 1994). Therefore, variability in climatic variables such as relative humidity, temperature, wind speed, and solar radiation due to the variation in surface characteristics can significantly affect precipitation formation. I analyzed the impact of geographical characteristics (elevation, freshwater areas, urbanization, and proximity to coast) on hydroclimatic variables (precipitation, pan evaporation, temperature, wind speed, relative humidity, and solar radiation) while conducting analyses on the effects of geographical and climatic factors on the long-term averages and trends of precipitation and pan evaporation.

Urbanization has a close relationship with precipitation. With respect to the effect of the modified sensible and latent heat on convective precipitation clouds in urbanized regions, most researchers have found that urban circulation is primarily enhanced by the 
increased sensible heat fluxes and surface roughness of urban regions, and that sensible heat has a larger effect than moisture in determining the location of initial cumulus activities and on the formation of convective clouds (Orville et al. 1981). Jauregui \& Romales (1996) found that wet season rainfall for an urban site exhibited a significant trend, suggesting an urban effect that increased with the size of the city. On the other hand, rainfall at a suburban (upwind) station that was apparently unaffected by urbanization remained unchanged. Urbanization is also known to have an effect on pan evaporation (Adebayo 1991, Jauregui \& Luyando 1998).

In addition, sensible heat flux, latent heat flux, and aerodynamic turbulence change according to geographical characteristics; the aerosols generated in urban areas also play an important role in precipitation and pan evaporation (Cohen et al. 2002, Roderick \& Farquhar 2002, Liu et al. 2004, Shepherd 2005, van den Heever \& Cotton 2007). Studies have been conducted on the effects of urbanization as well as geographical conditions including the sea, elevation, and freshwater areas (rivers, wetlands, lakes, and reservoirs) on precipitation and pan evaporation (Weisse \& Bois 2001, Sotillo et al. 2003, Oettli \& Camberlin 2005, Perry \& Konrad 2006, Kim \& Yim 2006, Mosaedi et al. 2007, Rim et al. 2009).

Changes in climatic factors have a direct impact on changes in precipitation or evaporation. Increasing precipitation leads to an increase in actual evaporation; therefore, reduced solar radiation and increased humidity in the air lead to decreased pan evaporation (potential evaporation; Brutsaert \& Parlange 1998). This implies that a complementary relationship can be applied to pan evaporation (potential evaporation) and actual evaporation (Golubev et al. 2001, Hobbins et al. 2004). Results obtained by Milly \& Dunne (2001) suggested that increases in cloudiness and precipitation over the Mississippi River Basin (USA) have led to increased evaporation but decreased potential evapotranspiration. Comparative analyses on correlations between pan evaporation and both precipitation and actual evaporation in 655 areas belonging to nonurban areas in the US have found that pan evaporation decreased at a time of increased precipitation, actual evaporation increased, solar radiation decreased, and relative humidity increased (Hobbins et al. 2004). In addition, a study conducted in 25 areas in the US found a complementary relationship between actual evaporation and pan evaporation depending on the changes in precipitation (Ramirez \& Hobbins 2005). On the other hand, many researchers (Cohen et al. 2002, Roderick \& Farquhar 2002, Liu et al. 2004) have reported results that differ from those of Brutsaert \& Parlange (1998). They argue that the reduced pan evaporation observed worldwide is due to reduced solar radiation caused by increasing aerosols in the wake of air contamination caused by increased cloudiness and urbanization.

Most of the studies carried out in the past have analyzed the changes in precipitation and pan evaporation using data obtained in limited areas. Other studies were conducted on changes in precipitation or evaporation according to climatic factors in various areas without the consideration of detailed data on geographical characteristics. However, changes in precipitation and evaporation have a close relationship not only with climatic factors but also with geographical characteristics. Accordingly, it is necessary to analyze the effect of geographical characteristics on precipitation or evaporation using detailed geographical information. Here I acquired geographical information using GIS analyses; the effect that the geographical characteristics have on climatic factors was analyzed using the acquired information. I also evaluated the effects of geographical and climatic factors on the long-term averages and trends of precipitation and pan evaporation.

\section{DATA AND METHODOLOGY}

\subsection{Study areas and geographical data}

In this study, 54 study areas located in South Korea were selected. Study areas were selected based on the usability of climatic data. A climatological station was centrally located in each study area having a $10 \mathrm{~km}$ radius, and the 54 study areas were located between Stn 1 in the north $\left(38^{\circ} 15^{\prime} N\right)$, Stn 26 in the south $\left(33^{\circ} 15^{\prime} \mathrm{N}\right)$, Stns 10 and 47 in the east $\left(129^{\circ} 25^{\prime} \mathrm{E}\right)$, Stn 22 in the west $\left(126^{\circ} 23^{\prime}\right.$ E, Fig. 1). South Korea is surrounded by the sea. Most cities and farms are situated on 2 fertile plains: the southwestern plain that covers the western half of South Korea, and the southern plain along the south coast. South Korea has a temperate climate with 4 distinct seasons; winters are cold and dry, whereas summers are hot and humid. Spring and autumn are mild. The specific geographical and topographical characteristics of the 54 study areas are listed in Table 1.

The following considerations were made in selecting study areas. (1) To examine the effect of urbanization at the observation station on hydroclimatological variables, the urbanization was calculated as the ratio of residential area to total area. (2) To examine the effect of the distance to coast on hydroclimatological variables, the proximity of the observation station to the sea was used. Only stations located on the coast were designated 'coastal'; all others were designated 'in- 


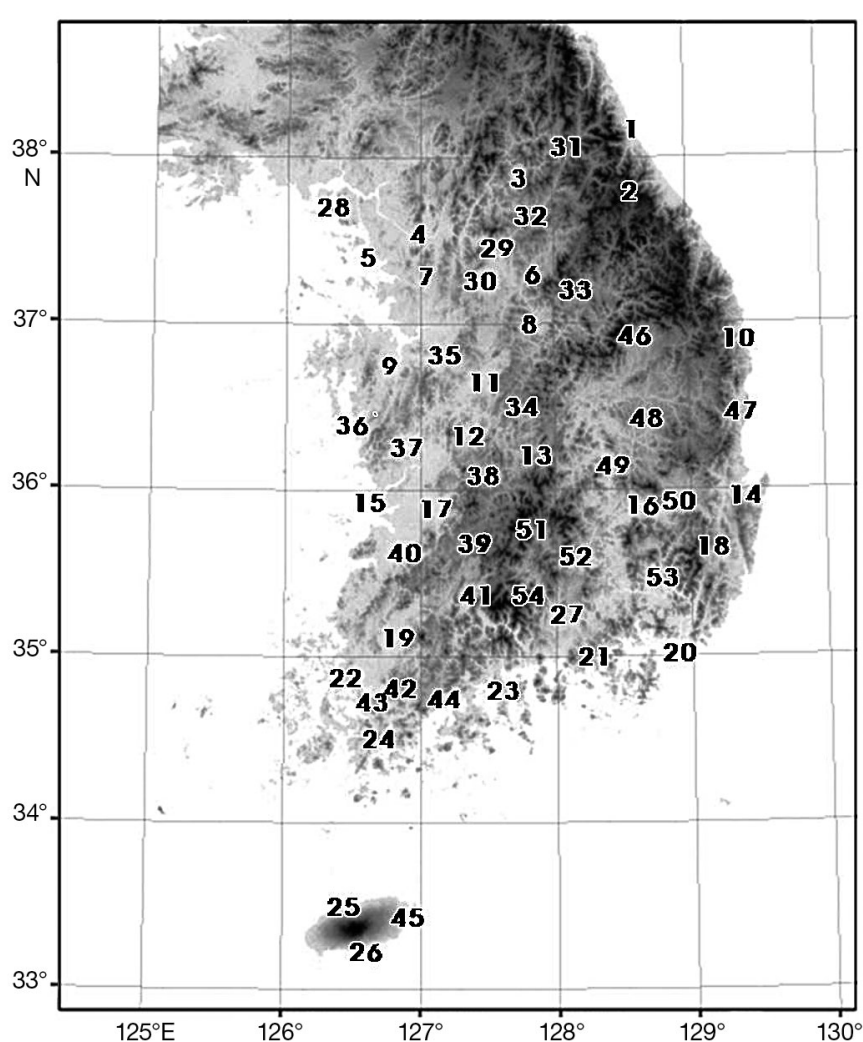

Fig. 1. Climatological stations. Shadings represent topography on the Korean peninsula. Station numbers refer to Table 1

land'. (3) I examined the effect of geographical conditions, such as the presence of lakes and marshes, plains, and mountains near the observation station, on hydroclimatological variables. To examine the effect of freshwater area at the observation station on hydroclimatological variables, the freshwater area (percentage) was calculated as the ratio freshwater area:total area. To classify the study areas, digital elevation model (DEM) data and a land cover map operated by the Ministry of Land, Transport, and Maritime Affairs (MLTM) were analyzed. DEM data for 2004 and land cover maps for 6 years $(1975,1980,1985,1990,1995$ and 2000) were analyzed. For 1975 and 1980, the data from Landsat Multi-spectral Scanner were used. For 1985, 1990, and 1995, data from Landsat Thematic Mapper and for 2000, data from Landsat Enhanced Thematic Mapper were used for analysis (Table 1).

For the 54 study areas selected, I conducted a GIS analysis of an area of $314 \mathrm{~km}^{2}$ around the observation station, i.e. the area within a $10 \mathrm{~km}$ radius centered at the observation station. I also analyzed land cover status and average elevation of the study area for 6 years $(1975,1980,1985,1990,1995$, and 2000); land cover maps for 1970 and 2004 were unavailable. The 54 study areas were evenly distributed along the coast and in- land throughout the Korean Peninsula (Fig. 1). In addition, the geographical characteristics of the study areas had an even distribution of plains, mountains, lakes, and marshes. Therefore, the selection of study areas was appropriate for the analysis of the hydroclimatic changes caused by geographical conditions.

\subsection{Climatic data}

For this study, climate data measured by the Korean Meteorological Administration (KMA) were obtained from 54 stations in Korea. The collected and analyzed climate data included the monthly average daily precipitation, temperature, wind speed, relative humidity, and sunshine duration. The data period used for this study was a 34 yr period from 1973 to 2006 (Fig. 2). Sunshine duration at the 54 weather stations was measured using a Jordan sunshine recorder until 1999; after this time, different sunshine recorders (i.e. ECO sunshine duration meter MS-093, bimetal sunshine recorder) were used. Therefore, to analyze the trend of solar radiation estimated from Eq. (1) below, sunshine duration data measured until 1999 were used for the 54 study areas. In the case of small-scale pan evaporation, data for only an $18 \mathrm{yr}$ period from 1973 to 1990 were available in the 54 study areas; therefore, pan evaporation data during this period were used for analyses. Average values of hydroclimatic data (precipitation, temperature, relative humidity, wind speed, and solar radiation) during the same period in the same study areas were also calculated for the analyses (Fig. 3). South Korea has 4 seasons with a wet summer from June to August, and a cold winter from December to February. Therefore, for the analyses of monthly climatic data, climatic data for the months of January, April, July, and October were collected and analyzed with respect to the seasonal effects. The following formula was used for calculating solar radiation, as suggested by Allen et al. (1998).

$$
\begin{gathered}
Q_{s}=\left(0.25+0.50 \frac{n}{N}\right) Q_{a} \\
Q_{a}=37.586 d_{r}\left[\omega_{s} \sin (\phi) \sin (\delta)+\cos (\phi) \cos (\delta) \sin \left(\omega_{s}\right)\right] \\
d_{r}=1+0.033 \cos \left[\frac{2 \pi}{365} J\right] \\
\delta=0.409 \sin \left[\frac{2 \pi}{365} J-1.39\right] \\
\omega_{s}=\frac{\pi}{2}-\arctan \left[\frac{-\tan (\phi) \tan (\delta)}{X^{0.5}}\right] \\
X=1-[\tan (\phi)]^{2}[\tan (\delta)]^{2} \\
X=0.00001 \quad \text { if } \quad X \leq 0
\end{gathered}
$$

where $Q_{S}$ is shortwave radiation $\left(\mathrm{MJ} \mathrm{m}^{-2} \mathrm{~d}^{-1}\right) ; Q_{a}$ is extraterrestrial radiation $\left(\mathrm{MJ} \mathrm{m}^{-2} \mathrm{~d}^{-1}\right) ; n$ is the actual 
Table 1. Geographical characteristics of 54 study areas. C: study station located near coast; I: study station located inland. 'Inland area of study station': area located inland. 'Freshwater area (\%)': ratio of freshwater area to total area $\left(314 \mathrm{~km}^{2}\right)$

\begin{tabular}{|c|c|c|c|c|c|c|c|c|c|}
\hline \multirow[t]{3}{*}{ Station (no.) } & \multirow{3}{*}{$\begin{array}{l}\text { Lat. } \\
(\mathrm{N})\end{array}$} & \multirow{3}{*}{$\begin{array}{l}\text { Long. } \\
\text { (E) }\end{array}$} & \multirow{3}{*}{$\begin{array}{c}\text { Avg. } \\
\text { elev. (m) }\end{array}$} & \multicolumn{6}{|c|}{$\longrightarrow$ Geographical classification } \\
\hline & & & & \multicolumn{2}{|c|}{ Geographical location } & \multicolumn{2}{|c|}{ Urbanization $(\%)$} & \multicolumn{2}{|c|}{ Freshwater area (\%) } \\
\hline & & & & $\begin{array}{c}\text { Coast } \\
\text { or } \\
\text { Inland }\end{array}$ & $\begin{array}{l}\text { Inland area of } \\
\text { study station } \\
\quad\left(\mathrm{km}^{2}\right)\end{array}$ & $\begin{array}{c}6 \mathrm{yr} \\
\text { mean }\end{array}$ & $\begin{array}{l}4 \text { yr } \\
\text { mean }\end{array}$ & $\begin{array}{c}6 \text { yr } \\
\text { mean }\end{array}$ & $\begin{array}{c}4 \mathrm{yr} \\
\text { mean }\end{array}$ \\
\hline Sokcho (1) & $38^{\circ} 15^{\prime}$ & $128^{\circ} 34^{\prime}$ & 115 & $\mathrm{C}$ & 165 & 3.5 & 2.2 & 0.7 & 0.7 \\
\hline Daegwallyeong (2) & $37^{\circ} 41^{\prime}$ & $128^{\circ} 46^{\prime}$ & 655 & $\mathrm{I}$ & 314 & 0.3 & 0.2 & 0.3 & 0.3 \\
\hline Chuncheon (3) & $37^{\circ} 54^{\prime}$ & $127^{\circ} 44^{\prime}$ & 213 & I & 314 & 5.6 & 4.4 & 6.7 & 6.7 \\
\hline Seoul (4) & $37^{\circ} 34^{\prime}$ & $126^{\circ} 58^{\prime}$ & 70 & I & 314 & 51.2 & 47.3 & 6.2 & 6.4 \\
\hline Incheon (5) & $37^{\circ} 28^{\prime}$ & $126^{\circ} 38^{\prime}$ & 26 & $\mathrm{C}$ & 193 & 36.7 & 29.3 & 13.7 & 18.8 \\
\hline Wonju (6) & $37^{\circ} 20^{\prime}$ & $127^{\circ} 57^{\prime}$ & 295 & I & 314 & 3.1 & 1.6 & 0.2 & 0.2 \\
\hline Suwon (7) & $37^{\circ} 16^{\prime}$ & $126^{\circ} 59^{\prime}$ & 73 & I & 314 & 13.0 & 8.8 & 1.2 & 1.3 \\
\hline Chungju (8) & $36^{\circ} 58^{\prime}$ & $127^{\circ} 57^{\prime}$ & 182 & I & 314 & 3.4 & 2.8 & 7.0 & 7.0 \\
\hline Seosan (9) & $36^{\circ} 46^{\prime}$ & $126^{\circ} 30^{\prime}$ & 53 & $\mathrm{I}$ & 314 & 3.7 & 2.6 & 2.5 & 3.1 \\
\hline Uljin (10) & $36^{\circ} 59^{\prime}$ & $129^{\circ} 25^{\prime}$ & 105 & $\mathrm{C}$ & 162 & 1.5 & 1.2 & 0.8 & 0.8 \\
\hline Cheongju (11) & $36^{\circ} 38^{\prime}$ & $127^{\circ} 27^{\prime}$ & 83 & I & 314 & 8.3 & 6.2 & 1.4 & 1.7 \\
\hline Daejeon (12) & $36^{\circ} 22^{\prime}$ & $127^{\circ} 22^{\prime}$ & 113 & I & 314 & 15.3 & 13.0 & 0.6 & 0.7 \\
\hline Chupungnyeong (13) & $36^{\circ} 13^{\prime}$ & $128^{\circ} 00^{\prime}$ & 318 & I & 314 & 0.4 & 0.3 & 0.3 & 0.3 \\
\hline Pohang (14) & $36^{\circ} 02^{\prime}$ & $129^{\circ} 23^{\prime}$ & 51 & $\mathrm{C}$ & 234 & 8.0 & 5.7 & 3.0 & 3.5 \\
\hline Gunsan (15) & $36^{\circ} 00^{\prime}$ & $126^{\circ} 45^{\prime}$ & 16 & $\mathrm{C}$ & 250 & 9.8 & 7.6 & 7.0 & 7.4 \\
\hline Daegu (16) & $35^{\circ} 53^{\prime}$ & $128^{\circ} 37^{\prime}$ & 111 & I & 314 & 17.7 & 14.5 & 1.3 & 1.3 \\
\hline Jeonju (17) & $35^{\circ} 49^{\prime}$ & $127^{\circ} 09^{\prime}$ & 107 & I & 314 & 8.8 & 7.6 & 1.0 & 1.0 \\
\hline Ulsan (18) & $35^{\circ} 33^{\prime}$ & $129^{\circ} 19^{\prime}$ & 78 & I & 314 & 4.7 & 2.3 & 1.7 & 1.9 \\
\hline Gwangju (19) & $35^{\circ} 10^{\prime}$ & $126^{\circ} 54^{\prime}$ & 85 & I & 314 & 13.6 & 10.3 & 1.3 & 1.3 \\
\hline Busan (20) & $35^{\circ} 06^{\prime}$ & $129^{\circ} 02^{\prime}$ & 86 & $\mathrm{C}$ & 197 & 32.5 & 29.1 & 6.3 & 7.3 \\
\hline Tongyeong (21) & $34^{\circ} 51^{\prime}$ & $128^{\circ} 26^{\prime}$ & 81 & $\mathrm{C}$ & 177 & 2.1 & 1.5 & 4.1 & 4.8 \\
\hline Mokpo (22) & $34^{\circ} 49^{\prime}$ & $126^{\circ} 23^{\prime}$ & 24 & $\mathrm{C}$ & 174 & 9.0 & 5.3 & 14.0 & 17.2 \\
\hline Yeosu (23) & $34^{\circ} 44^{\prime}$ & $127^{\circ} 45^{\prime}$ & 106 & $\mathrm{C}$ & 130 & 10.3 & 8.2 & 2.3 & 3.1 \\
\hline Wando (24) & $34^{\circ} 24^{\prime}$ & $126^{\circ} 43^{\prime}$ & 92 & $\mathrm{C}$ & 186 & 3.2 & 2.2 & 6.8 & 8.2 \\
\hline JeJu (25) & $33^{\circ} 31^{\prime}$ & $126^{\circ} 32^{\prime}$ & 177 & $\mathrm{C}$ & 166 & 10.9 & 7.4 & 0.4 & 0.5 \\
\hline Seogwipo (26) & $33^{\circ} 15^{\prime}$ & $126^{\circ} 34^{\prime}$ & 270 & $\mathrm{C}$ & 163 & 5.2 & 2.9 & 0.4 & 0.5 \\
\hline Jinju (27) & $35^{\circ} 12^{\prime}$ & $128^{\circ} 07^{\prime}$ & 72 & I & 314 & 3.0 & 2.4 & 2.2 & 2.1 \\
\hline Ganghwa (28) & $37^{\circ} 42^{\prime}$ & $126^{\circ} 27^{\prime}$ & 58 & $\mathrm{C}$ & 270 & 4.2 & 3.8 & 1.5 & 1.5 \\
\hline Yangpyeong (29) & $37^{\circ} 29^{\prime}$ & $127^{\circ} 30^{\prime}$ & 204 & I & 314 & 0.8 & 0.6 & 2.9 & 2.9 \\
\hline Icheon (30) & $37^{\circ} 16^{\prime}$ & $127^{\circ} 29^{\prime}$ & 80 & I & 314 & 2.6 & 1.6 & 0.0 & 0.0 \\
\hline Inje (31) & $38^{\circ} 03^{\prime}$ & $128^{\circ} 10^{\prime}$ & 488 & I & 314 & 1.0 & 0.7 & 2.1 & 2.7 \\
\hline Hongcheon (32) & $37^{\circ} 41^{\prime}$ & $127^{\circ} 53^{\prime}$ & 285 & I & 314 & 2.1 & 1.7 & 0.9 & 1.0 \\
\hline Jecheon (33) & $37^{\circ} 09^{\prime}$ & $128^{\circ} 12^{\prime}$ & 365 & I & 314 & 2.9 & 2.6 & 0.2 & 0.2 \\
\hline Boeun (34) & $36^{\circ} 29^{\prime}$ & $127^{\circ} 44^{\prime}$ & 270 & I & 314 & 1.9 & 1.5 & 0.4 & 0.4 \\
\hline Cheonan (35) & $36^{\circ} 47^{\prime}$ & $127^{\circ} 07^{\prime}$ & 87 & I & 314 & 5.6 & 4.3 & 0.7 & 0.7 \\
\hline Boryeong (36) & $36^{\circ} 19^{\prime}$ & $126^{\circ} 34^{\prime}$ & 82 & $\mathrm{C}$ & 216 & 5.7 & 5.0 & 4.3 & 5.0 \\
\hline Buyeo (37) & $36^{\circ} 16^{\prime}$ & $126^{\circ} 55^{\prime}$ & 44 & I & 314 & 4.4 & 3.1 & 2.9 & 2.9 \\
\hline Geumsan (38) & $36^{\circ} 06^{\prime}$ & $127^{\circ} 29^{\prime}$ & 254 & I & 314 & 3.0 & 2.4 & 0.4 & 0.4 \\
\hline Imsil (39) & $35^{\circ} 37^{\prime}$ & $127^{\circ} 17^{\prime}$ & 271 & I & 314 & 1.9 & 0.5 & 0.6 & 0.7 \\
\hline Jeongeup (40) & $35^{\circ} 34^{\prime}$ & $126^{\circ} 52^{\prime}$ & 123 & I & 314 & 4.0 & 3.4 & 0.6 & 0.6 \\
\hline Namwon (41) & $35^{\circ} 24^{\prime}$ & $127^{\circ} 20^{\prime}$ & 166 & I & 314 & 3.2 & 0.9 & 0.6 & 0.8 \\
\hline Jangheung (42) & $34^{\circ} 41^{\prime}$ & $126^{\circ} 55^{\prime}$ & 154 & I & 314 & 2.3 & 1.7 & 0.8 & 0.9 \\
\hline Haenam (43) & $34^{\circ} 33^{\prime}$ & $126^{\circ} 34^{\prime}$ & 69 & $\mathrm{I}$ & 314 & 2.6 & 2.3 & 8.4 & 10.9 \\
\hline Goheung (44) & $34^{\circ} 37^{\prime}$ & $127^{\circ} 17^{\prime}$ & 81 & $\mathrm{C}$ & 240 & 3.6 & 2.8 & 2.5 & 3.0 \\
\hline Seongsanpo (45) & $33^{\circ} 23^{\prime}$ & $126^{\circ} 53^{\prime}$ & 94 & $\mathrm{C}$ & 169 & 3.7 & 1.6 & 0.5 & 0.5 \\
\hline Yeongju (46) & $36^{\circ} 52^{\prime}$ & $128^{\circ} 31^{\prime}$ & 458 & $\mathrm{I}$ & 314 & 0.5 & 0.3 & 0.2 & 0.1 \\
\hline Yeongdeok (47) & $36^{\circ} 32^{\prime}$ & $129^{\circ} 25^{\prime}$ & 119 & $\mathrm{C}$ & 197 & 0.3 & 0.3 & 0.7 & 0.7 \\
\hline Uiseong (48) & $36^{\circ} 21^{\prime}$ & $128^{\circ} 41^{\prime}$ & 178 & $\mathrm{I}$ & 314 & 0.4 & 0.3 & 0.3 & 0.3 \\
\hline Gumi (49) & $36^{\circ} 08^{\prime}$ & $128^{\circ} 19^{\prime}$ & 132 & I & 314 & 2.2 & 1.4 & 1.6 & 1.7 \\
\hline Yeongcheon (50) & $35^{\circ} 58^{\prime}$ & $128^{\circ} 57^{\prime}$ & 125 & I & 314 & 0.7 & 0.4 & 0.9 & 0.8 \\
\hline Geochang (51) & $35^{\circ} 40^{\prime}$ & $127^{\circ} 55^{\prime}$ & 364 & I & 314 & 0.3 & 0.2 & 0.2 & 0.2 \\
\hline Hapcheon (52) & $35^{\circ} 34^{\prime}$ & $128^{\circ} 10^{\prime}$ & 151 & I & 314 & 0.2 & 0.1 & 1.5 & 1.5 \\
\hline Miryang (53) & $35^{\circ} 29^{\prime}$ & $128^{\circ} 45^{\prime}$ & 137 & I & 314 & 1.1 & 1.0 & 1.2 & 1.2 \\
\hline Sancheong (54) & $35^{\circ} 25^{\prime}$ & $127^{\circ} 53^{\prime}$ & 369 & I & 314 & 0.2 & 0.1 & 0.4 & 0.5 \\
\hline
\end{tabular}



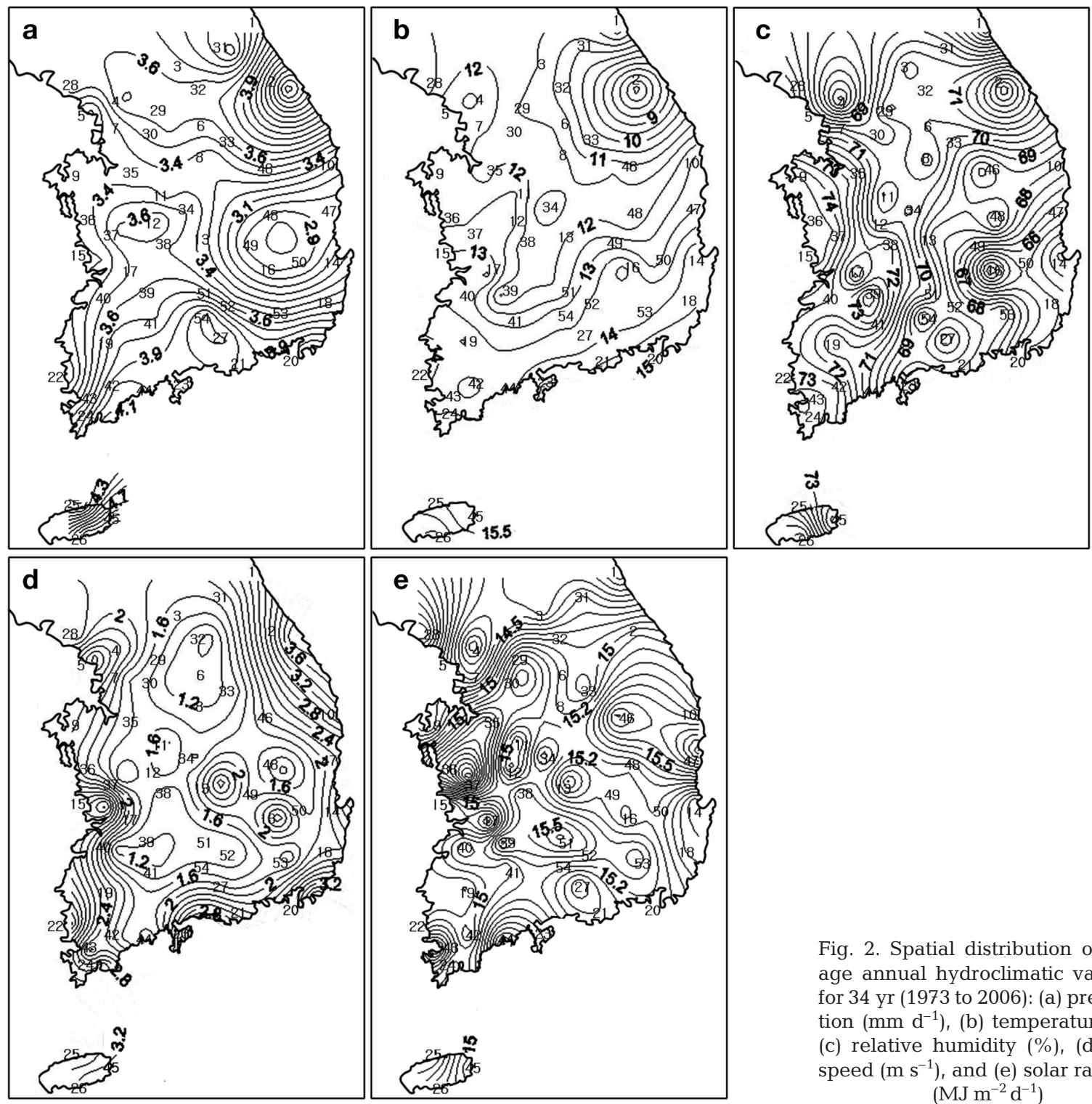

Fig. 2. Spatial distribution of average annual hydroclimatic variables for 34 yr (1973 to 2006): (a) precipitation $\left(\mathrm{mm} \mathrm{d}^{-1}\right)$, (b) temperature $\left({ }^{\circ} \mathrm{C}\right)$, (c) relative humidity (\%), (d) wind speed $\left(\mathrm{m} \mathrm{s}^{-1}\right)$, and (e) solar radiation $\left(\mathrm{MJ} \mathrm{m}^{-2} \mathrm{~d}^{-1}\right.$ )

duration of sunshine (h); $N$ is the maximum possible duration of sunshine or daylight hours; $n / N$ is the relative sunshine duration; $d_{r}$ is the inverse relative distance from Earth to the sun; $\delta$ is solar decimation (rad); $\omega_{s}$ is sunset hour angle (rad); $\phi$ is latitude (rad); and $J$ is the year-day.

\subsection{Analysis methods}

Mann-Kendall trend analyses were conducted to determine the trends of hydroclimatic variables (precipitation, pan evaporation, temperature, wind speed, relative humidity, and solar radiation); in order to analyze the relative importance that geographical factors have on the trends of hydroclimatic variables, multiple linear regression (MLR) analyses were performed. Furthermore, the effects of geographical factors on hydroclimatic variables were analyzed through MLR analyses. MLR analyses were conducted to identify the relative importance of geographical and climatic factors on the long-term averages of precipitation and pan evaporation. In this case, 2 different factors are considered to be the explanatory (independent) variables for the MLR analyses: geographical factors (average elevation, average freshwater area, average urbanization, and proximity to coast) and climatic factors (temperature, relative humidity, wind speed, and solar radiation). Multicollinearity between independent variables in the MLR equations was tested and was found to be low and therefore judged to be insignificant. Results were considered significant at $\alpha=0.05$. 


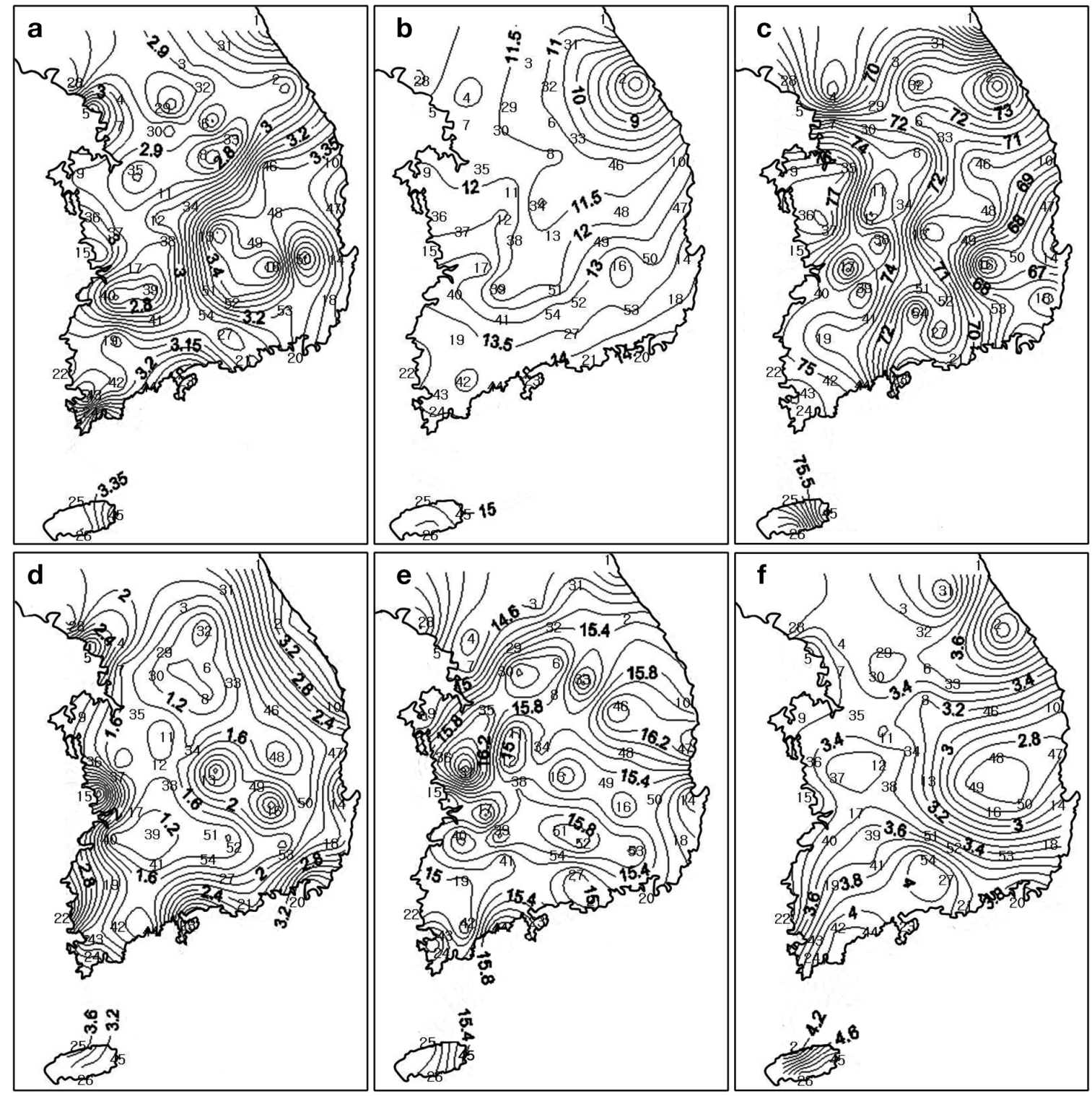

Fig. 3. Spatial distribution of average annual hydroclimatic variables for 18 yr (1973 to 1990): (a) pan evaporation (mm $\mathrm{d}^{-1}$ ), (b) temperature $\left({ }^{\circ} \mathrm{C}\right)$, (c) relative humidity $(\%)$, (d) wind speed $\left(\mathrm{m} \mathrm{s}^{-1}\right)$, (e) solar radiation $\left(\mathrm{MJ} \mathrm{m}^{-2} \mathrm{~d}^{-1}\right)$, and (f) precipitation $\left(\mathrm{mm} \mathrm{d} \mathrm{d}^{-1}\right)$

\section{RESULTS AND DISCUSSION}

\subsection{Geographical effects on climatic variables}

\subsubsection{Trends}

Relative to other geographical factors, the average urbanization had a greater effect on the annual temperature trend. As the urbanization increased, the temperature tended to increase (Table 2). In January, the proximity to the coast had a greater effect on the temperature trend than the other geographical factors: land areas that were farther inland had lower temperatures. In April, average urbanization, freshwater area, and proximity to coast had a significant effect on the temperature trend (2-tailed test). In July, no geographical factors had a significant effect on the temperature trend, and proximity to coast was the most important geographical factor. In October, the freshwater area and the urbanization had a significant effect on the temperature trend.

Urbanization had a greater effect relative to other geographical factors on the wind speed trend regardless of the season. As the urbanization of the study area increased, the wind speed decreased (Table 2). However, no geographical factors significantly affected the annual and monthly wind speed trends. 
Relative to other geographical factors, the urbanization had a greater effect on the annual relative humidity trend. As the urbanization of the study area increased, the relative humidity decreased (Table 2). In January and October, elevation had a greater effect on the relative humidity trend than other geographical factors: areas of higher elevation had higher relative humidity, reflecting an orographic effect. In April and July, proximity to coast had a greater effect on the relative humidity trend compared to other geographical factors. Land areas that were farther inland had lower relative humidity. However, no geographical factor had a significant effect on the relative humidity trend except for the urbanization in the case of the annual relative humidity trend.

The urbanization had a greater effect on the solar radiation trend relative to other geographical factors regardless of the season (Table 2). As the urbanization of a study area increased, the solar radiation increased. Furthermore, the urbanization had a significant effect on the annual and monthly solar radiation trends except in January.

Table 2. Relationships between geographical features and trends in temperature, wind speed, relative humidity and solar radiation. * Significance at $\alpha=0.05$ (2-tailed test); bold: geographic variables that have the greatest effect on climate variables. R: correlation coefficient of the multiple linear regression (MLR) equation. Parentheses: p-values; $\beta$ : standardized coefficient

\begin{tabular}{|c|c|c|c|c|c|c|}
\hline Period & $\mathrm{R}$ & $F$ & $\begin{array}{c}\text { Mean } \\
\text { elevation }\left(x_{1}\right) \\
\beta\end{array}$ & $\begin{array}{c}\text { Mean } \\
\text { freshwater area }\left(x_{2}\right) \\
\beta\end{array}$ & $\begin{array}{c}\text { Mean } \\
\text { urbanization }\left(x_{3}\right) \\
\beta\end{array}$ & $\begin{array}{c}\text { Proximity } \\
\text { to coast }\left(x_{4}\right) \\
\beta\end{array}$ \\
\hline \multicolumn{7}{|l|}{ Temperature } \\
\hline January & 0.610 & $\begin{array}{l}7.258 \\
(0.000)\end{array}$ & $\begin{array}{l}-0.027 \\
(0.834)\end{array}$ & $\begin{array}{c}-0.118 \\
(0.393)\end{array}$ & $\begin{array}{l}-0.071 \\
(0.593)\end{array}$ & $\begin{array}{c}-0.632^{*} \\
(0.000)\end{array}$ \\
\hline April & 0.504 & $\begin{array}{c}4.171 \\
(0.006)\end{array}$ & $\begin{array}{l}-0.051 \\
(0.721)\end{array}$ & $\begin{array}{c}-0.363^{*} \\
(0.019)\end{array}$ & $\begin{array}{l}0.396^{*} \\
(0.008)\end{array}$ & $\begin{array}{c}-0.349^{*} \\
(0.011)\end{array}$ \\
\hline July & 0.219 & $\begin{array}{c}0.619 \\
(0.651)\end{array}$ & $\begin{array}{l}-0.022 \\
(0.888)\end{array}$ & $\begin{array}{c}0.010 \\
(0.953)\end{array}$ & $\begin{array}{c}0.129 \\
(0.429)\end{array}$ & $\begin{array}{l}-0.137 \\
(0.367)\end{array}$ \\
\hline October & 0.462 & $\begin{array}{c}3.320 \\
(0.017)\end{array}$ & $\begin{array}{l}-0.159 \\
(0.276)\end{array}$ & $\begin{array}{c}-0.374^{*} \\
(0.019)\end{array}$ & $\begin{array}{l}0.370^{*} \\
(0.015)\end{array}$ & $\begin{array}{l}-0.226 \\
(0.104)\end{array}$ \\
\hline Year & 0.428 & $\begin{array}{c}2.747 \\
(0.039)\end{array}$ & $\begin{array}{r}0.021 \\
(0.888)\end{array}$ & $\begin{array}{l}-0.345 \\
(0.032)\end{array}$ & $\begin{array}{l}0.459^{*} \\
(0.004)\end{array}$ & $\begin{array}{l}-0.118 \\
(0.399)\end{array}$ \\
\hline \multicolumn{7}{|l|}{ Wind speed } \\
\hline January & 0.334 & $\begin{array}{c}1.533 \\
(0.207)\end{array}$ & $\begin{array}{c}0.030 \\
(0.848)\end{array}$ & $\begin{array}{c}0.011 \\
(0.948)\end{array}$ & $\begin{array}{l}-0.256 \\
(0.108)\end{array}$ & $\begin{array}{c}0.162 \\
(0.270)\end{array}$ \\
\hline April & 0.335 & $\begin{array}{l}1.550 \\
(0.203)\end{array}$ & $\begin{array}{c}0.052 \\
(0.734)\end{array}$ & $\begin{array}{c}0.053 \\
(0.745)\end{array}$ & $\begin{array}{l}-0.320 \\
(0.046)\end{array}$ & $\begin{array}{c}0.064 \\
(0.662)\end{array}$ \\
\hline July & 0.284 & $\begin{array}{l}1.075 \\
(0.379)\end{array}$ & $\begin{array}{l}-0.068 \\
(0.666)\end{array}$ & $\begin{array}{l}-0.099 \\
(0.555)\end{array}$ & $\begin{array}{c}-0.222 \\
(0.170)\end{array}$ & $\begin{array}{c}0.074 \\
(0.618)\end{array}$ \\
\hline October & 0.355 & $\begin{array}{c}1.764 \\
(0.151)\end{array}$ & $\begin{array}{l}-0.094 \\
(0.540)\end{array}$ & $\begin{array}{l}-0.078 \\
(0.633)\end{array}$ & $\begin{array}{l}-0.305 \\
(0.055)\end{array}$ & $\begin{array}{c}0.107 \\
(0.460)\end{array}$ \\
\hline Year & 0.359 & $\begin{array}{c}1.810 \\
(0.142)\end{array}$ & $\begin{array}{l}-0.056 \\
(0.713)\end{array}$ & $\begin{array}{l}-0.022 \\
(0.890)\end{array}$ & $\begin{array}{l}-0.302 \\
(0.057)\end{array}$ & $\begin{array}{c}0.166 \\
(0.254)\end{array}$ \\
\hline \multicolumn{7}{|l|}{ Relative humidity } \\
\hline January & 0.388 & $\begin{array}{c}2.175 \\
(0.086)\end{array}$ & $\begin{array}{c}0.318 \\
(0.039)\end{array}$ & $\begin{array}{c}0.101 \\
(0.528)\end{array}$ & $\begin{array}{l}-0.200 \\
(0.196)\end{array}$ & $\begin{array}{l}-0.006 \\
(0.968)\end{array}$ \\
\hline April & 0.226 & $\begin{array}{c}0.657 \\
(0.625)\end{array}$ & $\begin{array}{c}0.118 \\
(0.462)\end{array}$ & $\begin{array}{l}-0.054 \\
(0.751)\end{array}$ & $\begin{array}{c}0.001 \\
(0.997)\end{array}$ & $\begin{array}{l}-0.232 \\
(0.128)\end{array}$ \\
\hline July & 0.226 & $\begin{array}{c}0.657 \\
(0.625)\end{array}$ & $\begin{array}{c}0.118 \\
(0.462)\end{array}$ & $\begin{array}{l}-0.054 \\
(0.751)\end{array}$ & $\begin{array}{c}0.001 \\
(0.997)\end{array}$ & $\begin{array}{l}-0.232 \\
(0.128)\end{array}$ \\
\hline October & 0.294 & $\begin{array}{l}1.156 \\
(0.342)\end{array}$ & $\begin{array}{c}0.260 \\
(0.102)\end{array}$ & $\begin{array}{c}0.205 \\
(0.220)\end{array}$ & $\begin{array}{l}-0.146 \\
(0.364)\end{array}$ & $\begin{array}{l}-0.076 \\
(0.607)\end{array}$ \\
\hline Year & 0.387 & $\begin{array}{c}2.158 \\
(0.088)\end{array}$ & $\begin{array}{c}0.142 \\
(0.348)\end{array}$ & $\begin{array}{c}0.148 \\
(0.358)\end{array}$ & $\begin{array}{c}-0.372^{*} \\
(0.019)\end{array}$ & $\begin{array}{c}0.009 \\
(0.951)\end{array}$ \\
\hline \multicolumn{7}{|l|}{ Solar radiation } \\
\hline January & 0.406 & $\begin{array}{c}2.418 \\
(0.061)\end{array}$ & $\begin{array}{l}-0.090 \\
(0.549)\end{array}$ & $\begin{array}{l}-0.073 \\
(0.647)\end{array}$ & $\begin{array}{c}0.344 \\
(0.028)\end{array}$ & $\begin{array}{l}-0.148 \\
(0.296)\end{array}$ \\
\hline April & 0.544 & $\begin{array}{c}5.160 \\
(0.002)\end{array}$ & $\begin{array}{l}-0.074 \\
(0.589)\end{array}$ & $\begin{array}{c}0.024 \\
(0.870)\end{array}$ & $\begin{array}{l}0.438^{*} \\
(0.003)\end{array}$ & $\begin{array}{l}-0.169 \\
(0.196)\end{array}$ \\
\hline July & 0.631 & $\begin{array}{l}8.101 \\
(0.000)\end{array}$ & $\begin{array}{l}-0.202 \\
(0.117)\end{array}$ & $\begin{array}{l}-0.076 \\
(0.574)\end{array}$ & $\begin{array}{l}\mathbf{0 . 5 0 8}^{*} \\
(0.000)\end{array}$ & $\begin{array}{l}-0.168 \\
(0.166)\end{array}$ \\
\hline October & 0.596 & $\begin{array}{c}6.752 \\
(0.000)\end{array}$ & $\begin{array}{l}-0.121 \\
(0.359)\end{array}$ & $\begin{array}{l}-0.148 \\
(0.291)\end{array}$ & $\begin{array}{l}0.521^{*} \\
(0.000)\end{array}$ & $\begin{array}{l}-0.232 \\
(0.067)\end{array}$ \\
\hline Year & 0.685 & $\begin{array}{l}10.802 \\
(0.000)\end{array}$ & $\begin{array}{l}-0.124 \\
(0.301)\end{array}$ & $\begin{array}{l}-0.021 \\
(0.868)\end{array}$ & $\begin{array}{l}0.597^{*} \\
(0.000)\end{array}$ & $\begin{array}{l}-0.142 \\
(0.210)\end{array}$ \\
\hline
\end{tabular}


Table 3. Relationships between geographical features and long-term average temperature, wind speed, relative humidity, solar radiation, average precipitation and pan evaporation. * Significance at $\alpha=0.05$ (2-tailed test); bold: geographic variables that have the greatest effect. R: correlation coefficient of the multiple linear regression equation. Parentheses: $p$-values; $\beta$ : standardized coefficient

\begin{tabular}{|c|c|c|c|c|c|c|}
\hline Period & $\mathrm{R}$ & $F$ & $\begin{array}{c}\text { Mean } \\
\text { elevation }\left(x_{1}\right) \\
\beta\end{array}$ & $\begin{array}{c}\text { Mean } \\
\text { freshwater area }\left(x_{2}\right) \\
\beta\end{array}$ & $\begin{array}{c}\text { Mean } \\
\text { urbanization }\left(x_{3}\right) \\
\beta\end{array}$ & $\begin{array}{c}\text { Proximity } \\
\text { to coast }\left(x_{4}\right) \\
\beta\end{array}$ \\
\hline \multicolumn{7}{|l|}{ Temperature } \\
\hline January & 0.742 & $\begin{array}{l}15.032 \\
(0.000)\end{array}$ & $\begin{array}{c}-0.360^{*} \\
(0.002)\end{array}$ & $\begin{array}{l}-0.198 \\
(0.094)\end{array}$ & $\begin{array}{c}-0.023 \\
(0.835)\end{array}$ & $\begin{array}{c}-0.620^{*} \\
(0.000)\end{array}$ \\
\hline April & 0.606 & $\begin{array}{c}7.102 \\
(0.000)\end{array}$ & $\begin{array}{r}-0.518^{*} \\
(0.000)\end{array}$ & $\begin{array}{l}-0.256 \\
(0.069)\end{array}$ & $\begin{array}{c}0.162 \\
(0.226)\end{array}$ & $\begin{array}{r}-0.216 \\
(0.084)\end{array}$ \\
\hline July & 0.663 & $\begin{array}{c}9.624 \\
(0.000)\end{array}$ & $\begin{array}{c}-0.700^{*} \\
(0.000)\end{array}$ & $\begin{array}{l}-0.117 \\
(0.372)\end{array}$ & $\begin{array}{c}0.042 \\
(0.734)\end{array}$ & $\begin{array}{l}0.323^{*} \\
(0.007)\end{array}$ \\
\hline October & 0.789 & $\begin{array}{l}20.125 \\
(0.000)\end{array}$ & $\begin{array}{c}-0.448^{*} \\
(0.000)\end{array}$ & $\begin{array}{l}-0.113 \\
(0.294)\end{array}$ & $\begin{array}{c}0.091 \\
(0.375)\end{array}$ & $\begin{array}{c}-0.541^{*} \\
(0.000)\end{array}$ \\
\hline Year & 0.710 & $\begin{array}{l}12.468 \\
(0.000)\end{array}$ & $\begin{array}{c}-0.513^{*} \\
(0.000)\end{array}$ & $\begin{array}{l}-0.182 \\
(0.141)\end{array}$ & $\begin{array}{c}0.058 \\
(0.625)\end{array}$ & $\begin{array}{c}-0.419^{*} \\
(0.000)\end{array}$ \\
\hline \multicolumn{7}{|l|}{ Wind speed } \\
\hline January & 0.760 & $\begin{array}{l}16.721 \\
(0.000)\end{array}$ & $\begin{array}{l}0.328^{*} \\
(0.003)\end{array}$ & $\begin{array}{c}0.134 \\
(0.241)\end{array}$ & $\begin{array}{c}0.158 \\
(0.149)\end{array}$ & $\begin{array}{c}-0.726^{*} \\
(0.000)\end{array}$ \\
\hline April & 0.776 & $\begin{array}{l}18.452 \\
(0.000)\end{array}$ & $\begin{array}{l}0.276^{*} \\
(0.010)\end{array}$ & $\begin{array}{c}0.173 \\
(0.120)\end{array}$ & $\begin{array}{l}0.276^{*} \\
(0.011)\end{array}$ & $\begin{array}{c}-0.669^{*} \\
(0.000)\end{array}$ \\
\hline July & 0.774 & $\begin{array}{l}18.353 \\
(0.000)\end{array}$ & $\begin{array}{c}0.085 \\
(0.414)\end{array}$ & $\begin{array}{c}0.144 \\
(0.196)\end{array}$ & $\begin{array}{l}0.282^{*} \\
(0.010)\end{array}$ & $\begin{array}{c}-0.630^{*} \\
(0.000)\end{array}$ \\
\hline October & 0.824 & $\begin{array}{l}25.986 \\
(0.000)\end{array}$ & $\begin{array}{c}0.206 \\
(0.030)\end{array}$ & $\begin{array}{c}0.099 \\
(0.317)\end{array}$ & $\begin{array}{c}0.189 \\
(0.050)\end{array}$ & $\begin{array}{r}-0.787^{*} \\
(0.000)\end{array}$ \\
\hline Year & 0.798 & $\begin{array}{l}21.501 \\
(0.000)\end{array}$ & $\begin{array}{l}0.231^{*} \\
(0.023)\end{array}$ & $\begin{array}{c}0.137 \\
(0.196)\end{array}$ & $\begin{array}{c}0.230 \\
(0.026)\end{array}$ & $\begin{array}{r}-0.729^{*} \\
(0.000)\end{array}$ \\
\hline \multicolumn{7}{|l|}{ Relative humidity } \\
\hline January & 0.521 & $\begin{array}{c}4.555 \\
(0.003)\end{array}$ & $\begin{array}{c}0.005 \\
(0.970)\end{array}$ & $\begin{array}{c}0.301 \\
(0.047)\end{array}$ & $\begin{array}{c}-0.271 \\
(0.061)\end{array}$ & $\begin{array}{l}0.479^{*} \\
(0.001)\end{array}$ \\
\hline April & 0.611 & $\begin{array}{c}7.280 \\
(0.000)\end{array}$ & $\begin{array}{l}-0.257 \\
(0.052)\end{array}$ & $\begin{array}{c}0.262 \\
(0.062)\end{array}$ & $\begin{array}{r}-0.308^{*} \\
(0.023)\end{array}$ & $\begin{array}{r}-0.374^{*} \\
(0.003)\end{array}$ \\
\hline July & 0.656 & $\begin{array}{c}9.270 \\
(0.000)\end{array}$ & $\begin{array}{c}0.125 \\
(0.314)\end{array}$ & $\begin{array}{c}0.227 \\
(0.088)\end{array}$ & $\begin{array}{l}-0.088 \\
(0.485)\end{array}$ & $\begin{array}{c}-0.607^{*} \\
(0.000)\end{array}$ \\
\hline October & 0.764 & $\begin{array}{l}17.207 \\
(0.000)\end{array}$ & $\begin{array}{c}0.094 \\
(0.375)\end{array}$ & $\begin{array}{l}0.303^{*} \\
(0.009)\end{array}$ & $\begin{array}{c}-0.509^{*} \\
(0.000)\end{array}$ & $\begin{array}{l}0.581^{*} \\
(0.000)\end{array}$ \\
\hline Year & 0.424 & $\begin{array}{c}2.687 \\
(0.042)\end{array}$ & $\begin{array}{l}-0.001 \\
(0.996)\end{array}$ & $\begin{array}{l}0.381^{*} \\
(0.019)\end{array}$ & $\begin{array}{r}-0.428^{*} \\
(0.006)\end{array}$ & $\begin{array}{c}0.141 \\
(0.315)\end{array}$ \\
\hline \multicolumn{7}{|l|}{ Solar radiation } \\
\hline January & 0.220 & $\begin{array}{c}0.626 \\
(0.646)\end{array}$ & $\begin{array}{c}0.047 \\
(0.769)\end{array}$ & $\begin{array}{r}-0.406 \\
(0.785)\end{array}$ & $\begin{array}{l}-0.170 \\
(0.300)\end{array}$ & $\begin{array}{l}-0.120 \\
(0.430)\end{array}$ \\
\hline April & 0.507 & $\begin{array}{c}4.247 \\
(0.005)\end{array}$ & $\begin{array}{c}-0.034 \\
(0.812)\end{array}$ & $\begin{array}{l}-0.017 \\
(0.911)\end{array}$ & $\begin{array}{c}-0.383^{*} \\
(0.010)\end{array}$ & $\begin{array}{c}0.282 \\
(0.038)\end{array}$ \\
\hline July & 0.432 & $\begin{array}{c}2.810 \\
(0.035)\end{array}$ & $\begin{array}{l}-0.079 \\
(0.593)\end{array}$ & $\begin{array}{l}-0.007 \\
(0.963)\end{array}$ & $\begin{array}{c}-0.446^{*} \\
(0.004)\end{array}$ & $\begin{array}{c}0.028 \\
(0.842)\end{array}$ \\
\hline October & 0.381 & $\begin{array}{c}2.079 \\
(0.098)\end{array}$ & $\begin{array}{l}-0.242 \\
(0.115)\end{array}$ & $\begin{array}{l}-0.010 \\
(0.953)\end{array}$ & $\begin{array}{c}-0.262 \\
(0.095)\end{array}$ & $\begin{array}{l}-0.224 \\
(0.122)\end{array}$ \\
\hline Year & 0.388 & $\begin{array}{c}2.711 \\
(0.086)\end{array}$ & $\begin{array}{l}-0.096 \\
(0.527)\end{array}$ & $\begin{array}{c}0.041 \\
(0.798)\end{array}$ & $\begin{array}{c}-0.420^{*} \\
(0.008)\end{array}$ & $\begin{array}{c}0.047 \\
(0.743)\end{array}$ \\
\hline \multicolumn{7}{|l|}{ Precipitation } \\
\hline January & 0.649 & $\begin{array}{c}8.914 \\
(0.000)\end{array}$ & $\begin{array}{c}0.087 \\
(0.485)\end{array}$ & $\begin{array}{c}-0.353^{*} \\
(0.010)\end{array}$ & $\begin{array}{c}0.000 \\
(0.998)\end{array}$ & $\begin{array}{c}-0.669^{*} \\
(0.000)\end{array}$ \\
\hline April & 0.424 & $\begin{array}{c}2.681 \\
(0.042)\end{array}$ & $\begin{array}{c}0.033 \\
(0.823)\end{array}$ & $\begin{array}{r}-0.155 \\
(0.329)\end{array}$ & $\begin{array}{l}-0.010 \\
(0.948)\end{array}$ & $\begin{array}{r}-0.450^{*} \\
(0.002)\end{array}$ \\
\hline July & 0.558 & $\begin{array}{c}5.544 \\
(0.001)\end{array}$ & $\begin{array}{l}0.372^{*} \\
(0.008)\end{array}$ & $\begin{array}{c}0.034 \\
(0.812)\end{array}$ & $\begin{array}{c}0.318 \\
(0.025)\end{array}$ & $\begin{array}{l}0.346^{*} \\
(0.009)\end{array}$ \\
\hline October & 0.635 & $\begin{array}{c}8.258 \\
(0.000)\end{array}$ & $\begin{array}{l}0.342^{*} \\
(0.009)\end{array}$ & $\begin{array}{l}-0.289 \\
(0.036)\end{array}$ & $\begin{array}{c}0.094 \\
(0.466)\end{array}$ & $\begin{array}{c}-0.622^{*} \\
(0.000)\end{array}$ \\
\hline Year & 0.481 & $\begin{array}{c}3.696 \\
(0.010)\end{array}$ & $\begin{array}{c}0.326 \\
(0.027)\end{array}$ & $\begin{array}{c}-0.236 \\
(0.126)\end{array}$ & $\begin{array}{c}0.160 \\
(0.277)\end{array}$ & $\begin{array}{r}-0.426^{*} \\
(0.003)\end{array}$ \\
\hline Pan evaporation & & & & & & \\
\hline January & 0.682 & $\begin{array}{l}10.664 \\
(0.000)\end{array}$ & $\begin{array}{c}0.074 \\
(0.531)\end{array}$ & $\begin{array}{l}-0.224 \\
(0.081)\end{array}$ & $\begin{array}{c}0.006 \\
(0.963)\end{array}$ & $\begin{array}{c}-0.736^{*} \\
(0.000)\end{array}$ \\
\hline April & 0.326 & $\begin{array}{c}1.462 \\
(0.228)\end{array}$ & $\begin{array}{c}0.277 \\
(0.076)\end{array}$ & $\begin{array}{l}-0.147 \\
(0.369)\end{array}$ & $\begin{array}{c}0.033 \\
(0.829)\end{array}$ & $\begin{array}{l}-0.077 \\
(0.602)\end{array}$ \\
\hline July & 0.291 & $\begin{array}{c}1.132 \\
(0.352)\end{array}$ & $\begin{array}{c}-0.232 \\
(0.140)\end{array}$ & $\begin{array}{l}-0.134 \\
(0.419)\end{array}$ & $\begin{array}{c}0.011 \\
(0.943)\end{array}$ & $\begin{array}{l}-0.165 \\
(0.272)\end{array}$ \\
\hline October & 0.728 & $\begin{array}{l}13.834 \\
(0.000)\end{array}$ & $\begin{array}{c}0.030 \\
(0.785)\end{array}$ & $\begin{array}{l}-0.052 \\
(0.663)\end{array}$ & $\begin{array}{c}0.075 \\
(0.505)\end{array}$ & $\begin{array}{c}-0.741^{*} \\
(0.000)\end{array}$ \\
\hline Year & 0.530 & $\begin{array}{c}4.773 \\
(0.002)\end{array}$ & $\begin{array}{c}0.049 \\
(0.721)\end{array}$ & $\begin{array}{l}-0.159 \\
(0.280)\end{array}$ & $\begin{array}{c}0.063 \\
(0.652)\end{array}$ & $\begin{array}{c}-0.568^{*} \\
(0.000)\end{array}$ \\
\hline
\end{tabular}




\subsubsection{Long-term averages}

Relationships between geographical features and long-term temperature, wind speed, relative humidity, solar radiation, precipitation and pan evaporation are shown in Table 3. As an example, with regard to temperature, elevation (standardized coefficient $\beta=$ $-0.513)$ and proximity to coast $(\beta=-0.419)$ significantly affected annual temperature (2-tailed test). In January, proximity to the coast $(\beta=-0.620)$ and elevation $(\beta=$ -0.360) had a significant effect, and in April, the elevation $(\beta=-0.518)$ was the most significant factor. In July, elevation $(\beta=-0.700)$ and proximity to coast $(\beta=0.323)$ were the most significant factors, and in October, proximity to coast $(\beta=-0.541)$ and elevation $(\beta=-0.448)$ were significant factors.

\subsection{Geographical effects on trends in precipitation and pan evaporation}

The elevation and urbanization had a significant effect on the annual precipitation trend (2-tailed test), and they had a greater effect on the annual precipitation trend than freshwater area and proximity to coast (Table 4). Elevation had a greater effect on the annual precipitation trend. Complex topographical features, including the lifting effect of mountains, play important roles in precipitation formation. The interaction between topography and prevailing winds is crucial for precipitation formation over mountain areas. Urbanization increases roughness and can enhance surface convergence, and in addition to the dynamical and thermal effects of urbanization, aerosols also play important roles in precipitation formation.

The freshwater area and elevation had a significant effect on the precipitation trend in January. In April, elevation was the most significant geographical factor. In July, elevation and freshwater area had a significant effect on the precipitation trend, and elevation was the most significant factor. In October, elevation was a significant factor. Overall, among all geographical factors, elevation had the greatest effect on monthly and annual precipitation trends. The MLR equations for trends in daily precipitation per month and year were significant (2-tailed test), except for April.

For the annual pan evaporation trend, proximity to coast was the most important geographical factor (Table 4). In January, the urbanization was the most significant factor. In April, freshwater area was the most significant factor, and in July and October, proximity to coast was the most significant factor.

Table 4. Relative importance of geographical features with respect to trends in precipitation and pan evaporation. $y_{1}:$ January; $y_{2}$ : April $y_{3}$ : July; $y_{4}$ : October; $y_{5}$ : annual. Other details as in Table 2

\begin{tabular}{|c|c|c|c|c|c|c|}
\hline $\begin{array}{l}\text { Month / } \\
\text { MLR equation }\end{array}$ & $\mathrm{R}$ & $F$ & $\begin{array}{c}\text { Mean } \\
\text { elevation }\left(x_{1}\right) \\
\beta\end{array}$ & $\begin{array}{c}\text { Mean } \\
\text { freshwater area }\left(x_{2}\right) \\
\beta\end{array}$ & $\begin{array}{c}\text { Mean } \\
\text { urbanization }\left(x_{3}\right) \\
\beta\end{array}$ & $\begin{array}{c}\text { Proximity } \\
\text { to coast }\left(x_{4}\right) \\
\beta\end{array}$ \\
\hline \multicolumn{7}{|l|}{ Precipitation } \\
\hline $\begin{aligned} y_{1}= & 0.690+0.001542 x_{1}+0.07873 x_{2} \\
& -0.0158 x_{3}-0.00164 x_{4}\end{aligned}$ & 0.539 & $\begin{array}{c}5.012 \\
(0.002)\end{array}$ & $\begin{array}{l}0.389^{*} \\
(0.007)\end{array}$ & $\begin{array}{l}0.477^{*} \\
(0.002)\end{array}$ & $\begin{array}{l}-0.287 \\
(0.046)\end{array}$ & $\begin{array}{l}-0.192 \\
(0.146)\end{array}$ \\
\hline $\begin{aligned} y_{2}= & -1.077+0.002277 x_{1}-0.00370 x_{2} \\
& +0.008247 x_{3}-0.00163 x_{4}\end{aligned}$ & 0.437 & $\begin{array}{l}2.895 \\
(0.031)\end{array}$ & $\begin{array}{l}0.480^{*} \\
(0.002)\end{array}$ & $\begin{array}{l}-0.019 \\
(0.905)\end{array}$ & $\begin{array}{c}0.125 \\
(0.405)\end{array}$ & $\begin{array}{l}-0.159 \\
(0.255)\end{array}$ \\
\hline $\begin{aligned} y_{3}= & 0.386+0.001665 x_{1}+0.05968 x_{2} \\
& +0.001248 x_{3}+0.001081 x_{4}\end{aligned}$ & 0.506 & $\begin{array}{c}4.222 \\
(0.005)\end{array}$ & $\begin{array}{l}\mathbf{0 . 4 6 8}^{*} \\
(\mathbf{0 . 0 0 2})\end{array}$ & $\begin{array}{l}0.404^{*} \\
(0.009)\end{array}$ & $\begin{array}{c}0.025 \\
(0.860)\end{array}$ & $\begin{array}{c}0.141 \\
(0.294)\end{array}$ \\
\hline $\begin{aligned} y_{4}= & -1.166+0.002991 x_{1}+0.03686 x_{2} \\
& +0.02243 x_{3}-0.00168 x_{4}\end{aligned}$ & 0.472 & $\begin{array}{l}3.503 \\
(0.014)\end{array}$ & $\begin{array}{l}0^{-493 *} \\
(0.001)\end{array}$ & $\begin{array}{c}0.146 \\
(0.343)\end{array}$ & $\begin{array}{c}0.267 \\
(0.074)\end{array}$ & $\begin{array}{l}-0.129 \\
(0.347)\end{array}$ \\
\hline $\begin{aligned} y_{5}= & 0.249+0.003828 x_{1}+0.03152 x_{2} \\
& +0.03398 x_{3}+0.001063 x_{4}\end{aligned}$ & 0.658 & $\begin{array}{l}9.345 \\
(0.000)\end{array}$ & $\begin{array}{l}0.655^{*} \\
(0.000)\end{array}$ & $\begin{array}{c}0.130 \\
(0.325)\end{array}$ & $\begin{array}{l}0.420^{*} \\
(0.002)\end{array}$ & $\begin{array}{c}0.084 \\
(0.470)\end{array}$ \\
\hline \multicolumn{7}{|l|}{ Pan evaporation } \\
\hline $\begin{aligned} y_{1}= & 1.242-0.000259 x_{1}-0.0167 x_{2} \\
& -0.0419 x_{3}-0.00534 x_{4}\end{aligned}$ & 0.334 & $\begin{array}{l}1.540 \\
(0.205)\end{array}$ & $\begin{array}{l}-0.025 \\
(0.870)\end{array}$ & $\begin{array}{l}-0.048 \\
(0.768)\end{array}$ & $\begin{array}{l}-0.258 \\
(0.099)\end{array}$ & $\begin{array}{l}-0.239 \\
(0.110)\end{array}$ \\
\hline $\begin{aligned} y_{2}= & 1.112+0.0003655 x_{1}+0.04202 x_{2} \\
& -0.0185 x_{3}-0.00235 x_{4}\end{aligned}$ & 0.198 & $\begin{array}{c}0.499 \\
(0.736)\end{array}$ & $\begin{array}{c}0.040 \\
(0.801)\end{array}$ & $\begin{array}{c}0.139 \\
(0.415)\end{array}$ & $\begin{array}{l}-0.130 \\
(0.418)\end{array}$ & $\begin{array}{l}-0.120 \\
(0.435)\end{array}$ \\
\hline $\begin{aligned} y_{3}= & 0.448-0.000496 x_{1}-0.0112 x_{2} \\
& -0.0149 x_{3}-0.000443 x_{4}\end{aligned}$ & 0.327 & $\begin{array}{l}1.466 \\
(0.227)\end{array}$ & $\begin{array}{l}-0.073 \\
(0.636)\end{array}$ & $\begin{array}{l}-0.049 \\
(0.763)\end{array}$ & $\begin{array}{l}-0.140 \\
(0.368)\end{array}$ & $\begin{array}{l}-0.301 \\
(0.045)\end{array}$ \\
\hline $\begin{aligned} y_{4}= & 0.952+0.0003269 x_{1}-0.0146 x_{2} \\
& -0.00573 x_{3}-0.00159 x_{4}\end{aligned}$ & 0.110 & $\begin{array}{c}0.150 \\
(0.962)\end{array}$ & $\begin{array}{c}0.039 \\
(0.811)\end{array}$ & $\begin{array}{l}-0.052 \\
(0.764)\end{array}$ & $\begin{array}{l}-0.043 \\
(0.790)\end{array}$ & $\begin{array}{l}-0.087 \\
(0.576)\end{array}$ \\
\hline $\begin{aligned} y_{5}= & 0.02400+0.001661 x_{1}+0.03749 x_{2} \\
& -0.0295 x_{3}-0.00433 x_{4}\end{aligned}$ & 0.237 & $\begin{array}{c}0.732 \\
(0.575)\end{array}$ & $\begin{array}{c}0.133 \\
(0.400)\end{array}$ & $\begin{array}{c}0.090 \\
(0.592)\end{array}$ & $\begin{array}{l}-0.151 \\
(0.344)\end{array}$ & $\begin{array}{l}-0.161 \\
(0.291)\end{array}$ \\
\hline
\end{tabular}




\subsection{Geographical and climatic effects on precipitation and pan evaporation}

Relationships between geographical features and long-term average precipitation are shown in Table 3. Proximity to coast ( $\beta=-0.426$ ) had the most significant effect on annual precipitation (2-tailed test), and land areas that were farther inland had less precipitation. A study area located farther from the sea was affected less by winds with high humidity. At higher elevations ( $\beta=0.326)$, the observed precipitation was greater, reflecting the orographic effect. Study sites with larger freshwater area $(\beta=-0.236)$ had less precipitation, reflecting a low occurrence of convective precipitation upon a decrease in the sensible heat flux with an increase in the freshwater area. At the same time, study areas with a higher urbanization had greater realized precipitation, especially in July, reflecting a more frequent occurrence of convective precipitation with the increase in sensible heat flux and surface roughness of the study area. Proximity to coast had the greatest effect on monthly and annual precipitation relative to other geographical factors. In addition, areas that were farther inland had less precipitation, except in July. Areas with less urbanization showed higher precipitation in July, compared to other months.

Relationships between climatic variables and longterm precipitation are shown in Table 5. In annual analysis, relative humidity $(\beta=0.377)$ had a greater effect on precipitation than temperature $(\beta=0.199)$, wind speed $(\beta=0.193)$, and solar radiation $(\beta=-0.066)$.
Results indicate that the effects of climatic factors on monthly and annual precipitation varied.

Relationships between geographical features and long-term pan evaporation are shown in Table 3. Relative to other geographical factors, proximity to coast $(\beta$ $=-0.568$ ) had a significant effect on annual pan evaporation. Land areas that were farther inland showed less pan evaporation, whereas areas of higher elevation showed greater levels of pan evaporation. This reflects the different climatic conditions between inland and coastal areas. In particular, inland areas had a lower temperature than coastal areas except in July, and inland areas had a lower wind speed than coastal areas. In addition, study sites with larger freshwater area experienced less pan evaporation, reflecting the increase in relative humidity with an increase in freshwater area, whereas sites with higher urbanization showed greater pan evaporation. Results indicate that among all geographical factors, proximity to coast had the most important effect, except in April and July.

Relationships between climatic variables and longterm pan evaporation are shown in Table 5. In the annual analysis, wind speed $(\beta=0.474)$ had a greater effect on pan evaporation than temperature $(\beta=0.339)$, relative humidity $(\beta=-0.354)$, solar radiation $(\beta=$ $0.276)$, and precipitation $(\beta=-0.086)$. The most important climatic factor affecting pan evaporation was wind speed in July and October and relative humidity in January and April. Compared to other climatic variables, precipitation was not a significant factor regardless of the season.

Table 5. Relationships between climate variables and long-term average precipitation and pan evaporation. Other details as in Table 2

\begin{tabular}{|c|c|c|c|c|c|c|c|}
\hline Period & $\mathrm{R}$ & $\mathrm{F}$ & $\begin{array}{c}\text { Air } \\
\text { temperature } \\
\beta\end{array}$ & $\begin{array}{l}\text { Wind } \\
\text { speed } \\
\beta\end{array}$ & $\begin{array}{c}\text { Relative } \\
\text { humidity } \\
\beta\end{array}$ & $\begin{array}{c}\text { Solar } \\
\text { radiation } \\
\beta\end{array}$ & Precipitation \\
\hline \multicolumn{8}{|c|}{ Precipitation } \\
\hline January & 0.647 & $\begin{array}{c}8.801 \\
(0.000)\end{array}$ & $\begin{array}{l}0.472^{*} \\
(0.001)\end{array}$ & $\begin{array}{l}0.373^{*} \\
(0.007)\end{array}$ & $\begin{array}{c}0.223 \\
(0.130)\end{array}$ & $\begin{array}{l}-0.026 \\
(0.838)\end{array}$ & \\
\hline April & 0.598 & $\begin{array}{c}6.803 \\
(0.000)\end{array}$ & $\begin{array}{l}0.372^{*} \\
(0.005)\end{array}$ & $\begin{array}{l}-0.011 \\
(0.936)\end{array}$ & $\begin{array}{l}0.425^{*} \\
(0.001)\end{array}$ & $\begin{array}{l}-0.062 \\
(0.662)\end{array}$ & \\
\hline July & 0.587 & $\begin{array}{c}6.426 \\
(0.000)\end{array}$ & $\begin{array}{l}-0.013 \\
(0.920)\end{array}$ & $\begin{array}{c}-0.840 * \\
(0.000)\end{array}$ & $\begin{array}{l}0.494^{*} \\
(0.004)\end{array}$ & $\begin{array}{c}-0.350^{*} \\
(0.009)\end{array}$ & \\
\hline October & 0.626 & $\begin{array}{c}7.883 \\
(0.000)\end{array}$ & $\begin{array}{l}-0.209 \\
(0.230)\end{array}$ & $\begin{array}{l}0.773^{*} \\
(0.000)\end{array}$ & $\begin{array}{c}0.233 \\
(0.172)\end{array}$ & $\begin{array}{c}0.055 \\
(0.661)\end{array}$ & \\
\hline Year & 0.449 & $\begin{array}{c}3.094 \\
(0.024)\end{array}$ & $\begin{array}{c}0.199 \\
(0.157)\end{array}$ & $\begin{array}{c}0.193 \\
(0.178)\end{array}$ & $\begin{array}{l}0.377^{*} \\
(0.006)\end{array}$ & $\begin{array}{l}-0.066 \\
(0.629)\end{array}$ & \\
\hline \multicolumn{8}{|c|}{ Pan evaporation } \\
\hline January & 0.924 & $\begin{array}{l}55.918 \\
(0.000)\end{array}$ & $\begin{array}{l}0.354^{*} \\
(0.000)\end{array}$ & $\begin{array}{l}0.299^{*} \\
(0.000)\end{array}$ & $\begin{array}{r}-0.383^{*} \\
(0.000)\end{array}$ & $\begin{array}{l}0.253^{*} \\
(0.000)\end{array}$ & $\begin{array}{c}0.041 \\
(0.563)\end{array}$ \\
\hline April & 0.731 & $\begin{array}{l}11.037 \\
(0.000)\end{array}$ & $\begin{array}{c}0.116 \\
(0.343)\end{array}$ & $\begin{array}{l}0.387^{*} \\
(0.002)\end{array}$ & $\begin{array}{r}-0.638^{*} \\
(0.000)\end{array}$ & $\begin{array}{c}-0.366^{*} \\
(0.005)\end{array}$ & $\begin{array}{l}-0.084 \\
(0.519)\end{array}$ \\
\hline July & 0.616 & $\begin{array}{c}5.857 \\
(0.000)\end{array}$ & $\begin{array}{l}0.395^{*} \\
(0.002)\end{array}$ & $\begin{array}{l}0.497^{*} \\
(0.007)\end{array}$ & $\begin{array}{l}-0.250 \\
(0.098)\end{array}$ & $\begin{array}{c}0.276 \\
(0.054)\end{array}$ & $\begin{array}{l}-0.184 \\
(0.181)\end{array}$ \\
\hline October & 0.741 & $\begin{array}{l}27.485 \\
(0.000)\end{array}$ & $\begin{array}{l}0.331^{*} \\
(0.002)\end{array}$ & $\begin{array}{l}0.414^{*} \\
(0.001)\end{array}$ & $\begin{array}{r}-0.186 \\
(0.086)\end{array}$ & $\begin{array}{l}0.249^{*} \\
(0.004)\end{array}$ & $\begin{array}{c}0.078 \\
(0.389)\end{array}$ \\
\hline Year & 0.757 & $\begin{array}{l}12.866 \\
(0.000)\end{array}$ & $\begin{array}{l}0.339^{*} \\
(0.003)\end{array}$ & $\begin{array}{l}0.474^{*} \\
(0.000)\end{array}$ & $\begin{array}{c}-0.354^{*} \\
(0.002)\end{array}$ & $\begin{array}{l}0.276^{*} \\
(0.012)\end{array}$ & $\begin{array}{l}-0.086 \\
(0.433)\end{array}$ \\
\hline
\end{tabular}


Table 6. Relative importance of geographical and climatic factors with respect to precipitation and pan evaporation. $y_{1}$ : January; $y_{2}$ : April; $y_{3}$ : July; $y_{4}$ : October; $y_{5}$ : annual. The data were analyzed using stepwise regression. Other details as in Table 2

\begin{tabular}{|c|c|c|c|c|c|c|c|c|c|c|c|}
\hline \multirow{2}{*}{ MLR equation } & \multirow[t]{2}{*}{$\mathrm{R}$} & \multirow[t]{2}{*}{$F$} & \multicolumn{4}{|c|}{$\longrightarrow$ Geographical factors } & \multirow[b]{2}{*}{$\begin{array}{c}\text { Temp. } \\
\left(x_{5}\right) \\
\beta\end{array}$} & \multicolumn{3}{|c|}{ Climatic factors } & \multirow[b]{2}{*}{$\begin{array}{c}\text { Precip. } \\
\left(x_{9}\right) \\
\beta\end{array}$} \\
\hline & & & $\begin{array}{l}\text { Mean } \\
\text { Elev. } \\
\left(x_{1}\right) \\
\beta\end{array}$ & $\begin{array}{c}\text { Mean } \\
\text { freshwater } \\
\text { area }\left(x_{2}\right) \\
\beta\end{array}$ & $\begin{array}{l}\text { Mean } \\
\text { urbanization } \\
\text { rate }\left(x_{3}\right) \\
\beta\end{array}$ & $\begin{array}{l}\text { Proximity } \\
\text { to coast } \\
\left(x_{4}\right) \\
\beta\end{array}$ & & $\begin{array}{c}\text { Wind } \\
\text { speed } \\
\left(x_{6}\right) \\
\beta\end{array}$ & $\begin{array}{c}\text { Relative } \\
\text { humidity } \\
\left(x_{7}\right) \\
\beta\end{array}$ & $\begin{array}{c}\text { Solar } \\
\text { radiation } \\
\left(x_{8}\right) \\
\beta\end{array}$ & \\
\hline \multicolumn{12}{|l|}{ Pan evaporation } \\
\hline $\begin{aligned} y_{1}= & 1.664+0.0005467 x_{1} \\
& +0.06493 x_{5}+0.119 x_{6} \\
& -0.0233 x_{7}+0.124 x_{8}\end{aligned}$ & 0.935 & $\begin{array}{l}66.924 \\
(0.000)\end{array}$ & $\begin{array}{l}0.173^{*} \\
(0.005)\end{array}$ & - & - & - & $\begin{array}{l}0.473^{*} \\
(0.000)\end{array}$ & $\begin{array}{l}0.262^{*} \\
(0.000)\end{array}$ & $\begin{array}{r}-0.390^{*} \\
(0.000)\end{array}$ & $\begin{array}{l}0.219^{*} \\
(0.001)\end{array}$ & - \\
\hline$y_{2}=7.383-0.0518 x_{7}$ & 0.630 & $\begin{array}{l}34.181 \\
(0.000)\end{array}$ & & - & - & - & - & - & $\begin{array}{r}-0.630^{*} \\
(0.000)\end{array}$ & - & - \\
\hline $\begin{aligned} y_{3}= & 1.552+0.128 x_{5} \\
& -0.0791 x_{9}\end{aligned}$ & 0.524 & $\begin{array}{c}9.657 \\
(0.000)\end{array}$ & - & - & - & - & $\begin{array}{l}0.407^{*} \\
(0.001)\end{array}$ & - & - & - & $\begin{array}{r}-0.373^{*} \\
(0.003)\end{array}$ \\
\hline $\begin{aligned} y_{4}= & -1.088+0.000748 x_{1} \\
& +0.120 x_{5}+0.257 x_{6} \\
& +0.129 x_{8}\end{aligned}$ & 0.872 & $\begin{array}{l}38.903 \\
(0.000)\end{array}$ & $\begin{array}{l}0.251^{*} \\
(0.007)^{*}\end{array}$ & - & - & - & $\begin{array}{c}0.584 \\
(0.000)\end{array}$ & $\begin{array}{l}0.477^{*} \\
(0.000)\end{array}$ & - & $\begin{array}{l}0.202^{*} \\
(0.008)\end{array}$ & - \\
\hline $\begin{aligned} y_{5}= & 3.324-0.00667 x_{3} \\
& +0.05244 x_{5}+0.187 x_{6} \\
& -0.0332 x_{7}+0.08257 x_{8}\end{aligned}$ & 0.776 & $\begin{array}{l}14.568 \\
(0.000)\end{array}$ & - & - & $\begin{array}{l}-0.212 \\
(0.043)\end{array}$ & - & $\begin{array}{l}0.315^{*} \\
(0.002)\end{array}$ & $\begin{array}{l}0.515^{*} \\
(0.000)\end{array}$ & $\begin{array}{r}-0.424^{*} \\
(0.000)\end{array}$ & $\begin{array}{c}0.232 \\
(0.030)\end{array}$ & - \\
\hline \multicolumn{12}{|c|}{ Precipitation } \\
\hline $\begin{aligned} y_{1}= & 0.05224-0.0570 x_{2} \\
& -0.00199 x_{4}+0.04911 x_{5} \\
& +0.181 x_{6}+0.02062 x_{7}\end{aligned}$ & 0.773 & $\begin{array}{l}14.211 \\
(0.000)\end{array}$ & - & $\begin{array}{c}-0.449^{*} \\
(0.000)\end{array}$ & - & $\begin{array}{l}-0.302 \\
(0.056)\end{array}$ & $\begin{array}{l}0.359^{*} \\
(0.006)\end{array}$ & $\begin{array}{l}0.406^{*} \\
(0.004)\end{array}$ & $\begin{array}{l}0.346^{*} \\
(0.002)\end{array}$ & - & - \\
\hline $\begin{aligned} y_{2}= & -10.347+0.003372 x_{1} \\
& +0.442 x_{5}+0.120 x_{7}\end{aligned}$ & 0.734 & $\begin{array}{l}19.466 \\
(0.000)\end{array}$ & $\begin{array}{l}0.559^{*} \\
(0.000)\end{array}$ & - & - & - & $\begin{array}{l}0^{688}{ }^{*} \\
(0.000)\end{array}$ & $\begin{array}{l}- \\
-\end{array}$ & $\begin{array}{l}0.619^{*} \\
(0.000)\end{array}$ & - & - \\
\hline $\begin{aligned} y_{3}= & -18.997+0.003979 x_{1} \\
& +0.09305 x_{3}+0.00633 x_{4} \\
& -1.883 x_{6}+0.344 x_{7}\end{aligned}$ & 0.785 & $\begin{array}{l}15.403 \\
(0.000)\end{array}$ & $\begin{array}{l}0.355^{*} \\
(0.001)\end{array}$ & - & $\begin{array}{l}0.601^{*} \\
(0.000)\end{array}$ & $\begin{array}{l}0.262) \\
(0.061)\end{array}$ & - & $\begin{aligned}-0.795^{*} \\
(0.000)\end{aligned}$ & $\begin{array}{l}0.686^{*} \\
(0.000)\end{array}$ & - & - \\
\hline $\begin{aligned} y_{4}= & -2.915-0.0674 x_{2} \\
& +0.618 x_{6}+0.05634 x_{7}\end{aligned}$ & $\begin{array}{c}0.741 \\
(0.000)\end{array}$ & $\begin{array}{l}20.302 \\
(0.000)\end{array}$ & - & $\begin{array}{c}-0.449^{*} \\
(0.000)\end{array}$ & - & - & - & $\begin{array}{l}\mathrm{0.915}^{*} \\
(0.000)\end{array}$ & $\begin{array}{l}0.383^{*} \\
(0.002)\end{array}$ & - & - \\
\hline $\begin{aligned} y_{5}= & -2.716+0.002155 x_{1} \\
& -0.00185 x_{4}+0.152 x_{5} \\
& +0.06456 x_{7}\end{aligned}$ & 0.644 & $\begin{array}{c}8.702 \\
(0.000)\end{array}$ & $\begin{array}{l}0.580^{*} \\
(0.000)\end{array}$ & - & - & $\begin{array}{l}-0.231 \\
(0.079)\end{array}$ & $\begin{array}{l}0.499^{*} \\
(0.002)\end{array}$ & - & $\begin{array}{l}0.404^{*} \\
(0.001)\end{array}$ & - & - \\
\hline
\end{tabular}

\subsection{Relative importance of geographical and} climatic factors on precipitation and pan evaporation

On the basis of a stepwise MLR analysis of geographical factors, elevation and proximity to coast were included in an MLR equation for annual precipitation (Table 6). From among all climatic factors, temperature and relative humidity were included. Accordingly, I found that areas with higher elevation, a geographical factor, had higher annual precipitation, whereas land areas that were farther inland had less annual precipitation. In addition, sites with higher temperature and relative humidity, which are climatic factors, had higher annual precipitation.

With regard to monthly precipitation, not only geographical factors but also climatic factors seemed to have a great effect. In January, freshwater area, proximity to coast, temperature, wind speed, and relative humidity were included in the MLR equation. In April, elevation, temperature, and relative humidity were included. In July, elevation, urbanization, and proximity to coast were included as the geographical factors, and wind speed and relative humidity were included as the climatic factors. In October, freshwater area, wind speed, and relative humidity were included.

With regard to annual pan evaporation, the urbanization was included in the MLR equation as a geographical factor, and temperature, wind speed, relative humidity, and solar radiation were included as the climatic factors (Table 6). Accordingly, areas with a higher urbanization, a geographical factor, had greater annual pan evaporation. In terms of climatic factors, wind speed was the most important factor, followed by relative humidity, temperature, and solar radiation.

For January, pan evaporation, elevation, temperature, wind speed, relative humidity, and solar radiation were included in the MLR equation. For April, only relative humidity was included. For July, temperature and precipitation were included. For October, elevation, temperature, wind speed, and solar radiation were included. Overall, climatic factors appeared to have a greater effect on annual and monthly pan evaporation than geographical factors. 


\section{CONCLUSIONS}

An MLR analysis and a Mann-Kendall trend analysis were conducted to analyze the impact of geographical factors (elevation, freshwater area, urbanization, and proximity to coast) and climatic factors (temperature, wind speed, relative humidity, and solar radiation) on the long-term averages and trends of precipitation and pan evaporation. According to the study results, in terms of annual and monthly precipitation, proximity to coast had the most significant effect on precipitation, and sites that were farther inland had greater precipitation, except in July. Compared to other months, areas with greater urbanization had significantly higher precipitation in July.

Compared to other geographical factors, proximity to coast had a greater effect on annual pan evaporation. Land areas that were farther inland had less pan evaporation, whereas sites at higher elevation had greater pan evaporation. In addition, sites with a larger freshwater area had less pan evaporation, whereas sites with higher urbanization had greater pan evaporation. Proximity to coast had the most significant effect on monthly pan evaporation, except in April and July.

Stepwise MLR analyses were used to identify the relative importance of geographical and climatic factors on precipitation and pan evaporation. As elevation, a geographical factor, increased, annual precipitation increased. However, areas farther inland experienced less precipitation. Areas with higher temperature and relative humidity had greater annual precipitation. Geographical as well as climatic factors appeared to have a great effect on monthly precipitation. Climatic factors appeared to have a more significant effect than geographical factors on annual and monthly pan evaporation. Elevation had a greater effect than other geographical factors on annual and monthly precipitation trends. With regard to the annual pan evaporation trend, proximity to coast was the most significant factor. In addition, the monthly pan evaporation trend was affected differently by geographical factors depending on the season.

\section{LITERATURE CITED}

Adebayo Y (1991) Heat island in a humid tropical city and its relationship with potential evaporation. Theor Appl Climatol 43:137-147

Allen RG, Pereira LS, Raes D, Smith M (1998) Crop evapotranspiration: guidelines for computing crop requirements. Irrigation and drainage paper No. 56. FAO, Rome

Brutsaert W, Parlange MB (1998) Hydrological cycle explains the evaporation paradox. Nature 396:30

Cohen S, Ianetz A, Stanhill G (2002) Evaporative climate changes at Bet Dagan, Israel, 1964-1998. Agric For Meteorol 111:83-91
Collins D, Avissar R (1994) An evaluation with the Fourier amplitude sensitivity test (FAST) of which land-surface parameters are of great importance for atmospheric modeling. J Clim 7:681-703

Golubev VS, Lawrimore JH, Groisman PY, Speranskaya NA and others (2001) Evaporation changes over the contiguous United States and the former USSR: a reassessment. Geophys Res Lett 28:2665-2668

$>$ Hobbins MT, Ramirez JA, Brown TC (2004) Trends in pan evaporation and actual evapotranspiration across the conterminous U.S.: paradoxical or complementary? Geophys Res Lett 30:L13503

IPCC (2007) Climate change 2007: the physical science basis. Contribution of Working Group I to the Fourth Assessment Report of the Intergovernmental Panel on Climate Change. Cambridge University Press, Cambridge

Jauregui E, Luyando E (1998) Long-term association between pan evaporation and the urban heat island in Mexico City. Atmosfera 11:45-60

Jauregui E, Romales E (1996) Urban effects on convective precipitation in Mexico City. Atmos Environ 30: 3383-3389

Kim GS, Yim TK (2006) Analysis of pan evaporation data from 1973 to 2004 in South Korea. J Korean Soc Civil Eng 26(6B):583-596

Liu B, Xu M, Henderson M, Gong W (2004) A spatial analysis of pan evaporation trends in China, 1955-2000. J Geophys Res 109(D15):D15102. doi:10.1029/2004JD004511

Milly PCD, Dunne KA (2001) Trends in evaporation and surface cooling in the Mississippi River basin. Geophys Res Lett 28:1219-1222

Mosaedi A, Sharifan H, Shahabi M (2007) Effects of topography on maximum daily precipitation in Golestan province (Iran). Geophys Res Abstr 9:02396

Oettli P, Camberlin P (2005) Influence of topography on monthly rainfall distribution over East Africa. Clim Res 28: 199-212

> Orville HD, Eckhoff PA, Peak JE, Hirsch JH, Kopp FJ (1981) Numerical simulation of the effects of cooling tower complexes on clouds and severe storms. Atmos Environ 15: 823-835

Perry LB, Konrad CE (2006) Relationships between NW flow snowfall and topography in the Southern Appalachians, USA. Clim Res 32:35-47

$>$ Ramirez JA, Hobbins MT (2005) Observational evidence of the complementary relationship in regional evaporation lends strong support for Bouchet's hypothesis. Geophys Res Lett 32:L15401. doi:10.1029/2005GL023549

Rim CS, Yoon SE, Song JI (2009) Evaluation of equations for estimating pan evaporation considering regional characteristics. J Korean Soc Civil Eng 29(1B):47-62

> Roderick ML, Farquhar GD (2002) The cause of decreased pan evaporation over the past 50 years. Science 298: $1410-1411$

> Shepherd JM (2005) A review of current investigations of urban-induced rainfall and recommendations for the future. Earth Interact 9:1-27

Sotillo MG, Ramis C, Romero R, Alonso S, Homar V (2003) Role of orography in the spatial distribution of precipitation over the Spanish Mediterranean zone. Clim Res 23: $247-261$

van den Heever SC, Cotton WR (2007) Urban aerosol impacts on downwind convective storms. J Appl Meteorol 46: $828-850$

Weisse AK, Bois P (2001) Topographic effects on statistical characteristics of heavy rainfall and mapping in the French Alps. J Appl Meteorol 40:720-740 